\title{
ReaR

\section{Impacto de la modalidad de carga de fluidos en el diagnóstico de la capacidad de respuesta a volumen}

Artículo original: Toscani L, Aya HD, Antonakaki D, Bastoni D, Watson X, Arulkumaran N, et al. What is the impact of the fluid challenge technique on diagnosis of fluid responsiveness? A systematic review and meta-analysis. Crit Care. 2017 Aug 4;21(1):207. doi: 10.1186/s13054-017-1796-9. (PubMed) ( $\underline{\text { HTML) }}$ (HTML2) (PDF)

Macías Pingarrón JP, Torrado-Criado MD, García Guerrero J, Sánchez Espinosa F.

Complejo Hospitalario Universitario de Badajoz.

\section{Resumen}

El objetivo de este estudio fue describir la "carga de volumen" y evaluar la diferencia en la proporción de "respondedores" (PR) según el tipo de fluido, el volumen, la duración de la infusión y el momento de la evaluación.

Métodos: Se realizaron búsquedas en MEDLINE y Embase para estudios que utilizan la carga de volumen como una prueba de precarga cardíaca con una descripción de la técnica, una definición reportada de capacidad de respuesta de fluidos y PR. El resultado primario fue la PR media, según el volumen de líquido, el tipo de líquidos, la tasa de infusión y el tiempo de evaluación.

Resultados: se incluyeron un total de 85 estudios (3601 pacientes) en el análisis. Las PR fueron 54.4\% (IC 95\% 46.9-62.7) donde se administró $<500 \mathrm{ml}, 57.2 \%$ (IC 95\% 52.9-61.0) donde se administraron 500 $\mathrm{ml} \mathrm{y} 60.5 \%$ (IC 95\% 35.9-79.2) donde $>500 \mathrm{ml}$ fue administrado $(\mathrm{p}=0.71)$. El PR no se vio afectado por el tipo de fluido. La RP fue similar entre los pacientes a los que se administró una carga de volumen durante $<15$ minutos (59.2\%, IC 95\% 54.2-64.1) y durante $15-30$ minutos (57.7\%, IC 95\% 52.4$62.4, p=1$ ). Cuando el tiempo de infusión fue $\geq 30$ minutos, hubo una PR menor de $49.9 \%$ (IC 95\% 45.6$54, \mathrm{p}=0.04)$. La respuesta se evaluó al final de la exposición a los fluidos, entre 1 y 10 minutos, y $>10$ minutos después de la exposición a los fluidos. Las proporciones de respondedores fueron 53.9\%, 57.7\% y $52.3 \%$, respectivamente $(\mathrm{p}=0.47)$.

Conclusiones: el PR disminuye con un tiempo de infusión largo. Es deseable estandarizar la carga de volumen.

\section{Introducción}

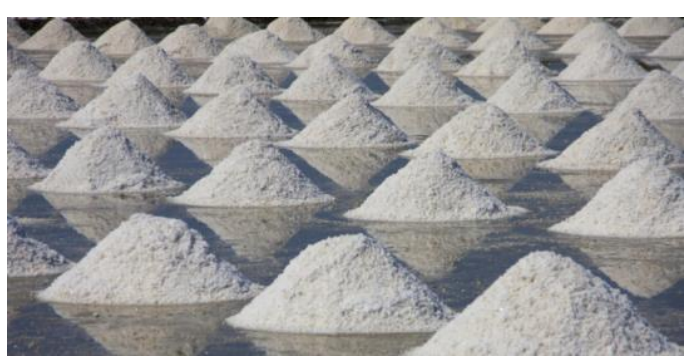

El objetivo de este estudio fue describir la "carga de volumen" y evaluar la diferencia en la proporción de "respondedores" (PR) según el tipo de fluido, el volumen, la duración de la infusión y el momento de la evaluación.

Métodos: Se realizaron búsquedas en MEDLINE y Embase para estudios que utilizan la carga de volumen como una prueba de precarga cardíaca con una descripción de la técnica, una definición reportada de capacidad de respuesta de fluidos y PR. El resultado primario fue la PR media, según el volumen de líquido, el tipo de líquidos, la tasa de infusión y el tiempo de evaluación.

Resultados: se incluyeron un total de 85 estudios (3601 pacientes) en el análisis. 
Las PR fueron 54.4\% (IC 95\% 46.962.7) donde se administró $<500 \mathrm{ml}$, $57.2 \%$ (IC 95\% 52.9-61.0) donde se administraron $500 \mathrm{ml}$ y $60.5 \%$ (IC 95\% 35.9-79.2) donde $>500 \mathrm{ml}$ fue administrado $(p=0.71)$. El PR no se vio afectado por el tipo de fluido. La RP fue similar entre los pacientes a los que se administró una carga de volumen durante $<15$ minutos $(59.2 \%$, IC 95\% 54.2-64.1) y durante 15-30 minutos $(57.7 \%$, IC 95\% 52.4-62.4, $\mathrm{p}=$ 1). Cuando el tiempo de infusión fue $\geq 30$ minutos, hubo una PR menor de $49.9 \%$ (IC 95\% 45.6-54, $\mathrm{p}=0.04$ ). $\mathrm{La}$ respuesta se evaluó al final de la exposición a los fluidos, entre 1 y 10 minutos, $\mathrm{y}>10$ minutos después de la exposición a los fluidos. Las proporciones de respondedores fueron $53.9 \%, \quad 57.7 \%$ y $52.3 \%$, respectivamente $(\mathrm{p}=0.47)$.

Conclusiones: el PR disminuye con un tiempo de infusión largo. Es deseable estandarizar la carga de volumen.

Las terapias basadas en la administración de líquidos intravenosos, son las más comúnmente administradas a pacientes críticamente enfermos (1). El objetivo de la expansión del volumen es aumentar el gasto cardíaco $(\mathrm{CO})$.

Una forma de evaluar la capacidad de respuesta de los fluidos y guiar su administración en pacientes críticos, es realizar una carga de volumen. Esto implica la infusión de una cantidad específica de líquido intravenoso para evaluar la reserva de precarga ventricular y los consiguientes efectos hemodinámicos sistémicos (2). El volumen de líquido infundido debe ser suficiente para aumentar el volumen diastólico del ventrículo derecho $\mathrm{y}$, posteriormente, el volumen sistólico (SV) (3). La respuesta a los fluidos se define convencionalmente como un aumento de al menos un $10 \%$ a $15 \%$ en el SV (4-5). Los pacientes que alcanzan este umbral se consideran "respondedores a volumen" (6).

Diferentes estudios clínicos han demostrado que aproximadamente el $50 \%$ de los pacientes críticamente enfermos, que se considera que tienen un $\mathrm{CO}$ inadecuado son respondedores a volumen.

La respuesta a la administración de los fluidos depende a su vez de la interacción dinámica entre el volumen intravascular, el tono vascular y la función ventricular. Además, la capacidad de respuesta a los fluidos también puede estar condicionada por las particularidades de la carga de volumen, incluidos el tipo, el volumen de fluido, así como la velocidad de administración.

El uso de diferentes métodos para estimar SV es un factor de confusión adicional.

El objetivo de este estudio fue describir las diferentes técnicas de carga de volumen utilizadas en ensayos clínicos, mediante la evaluación de la sensibilidad a los fluidos y analizar la proporción de pacientes considerados "fluidos-sensibles", que varía según la técnica utilizada.

\section{Método}

Se incluyeron los estudios publicados como artículos de texto completo, en inglés $y$ en revista indexada que cumplían los siguientes criterios:

-Uso de una carga de volumen como prueba de precarga cardíaca o como parte de un algoritmo clínico. -Realizados en Unidad de Cuidados Intensivos (UCI) o quirófano con pacientes adultos.

-Descripción completa de la carga de volumen (volumen, velocidad de 
infusión, tipo de líquido utilizado y momento de la evaluación de la respuesta hemodinámica).

-Definición clara de respuesta a los fluidos.

-Presentación clara del porcentaje de respondedores y no respondedores a la carga de volumen.

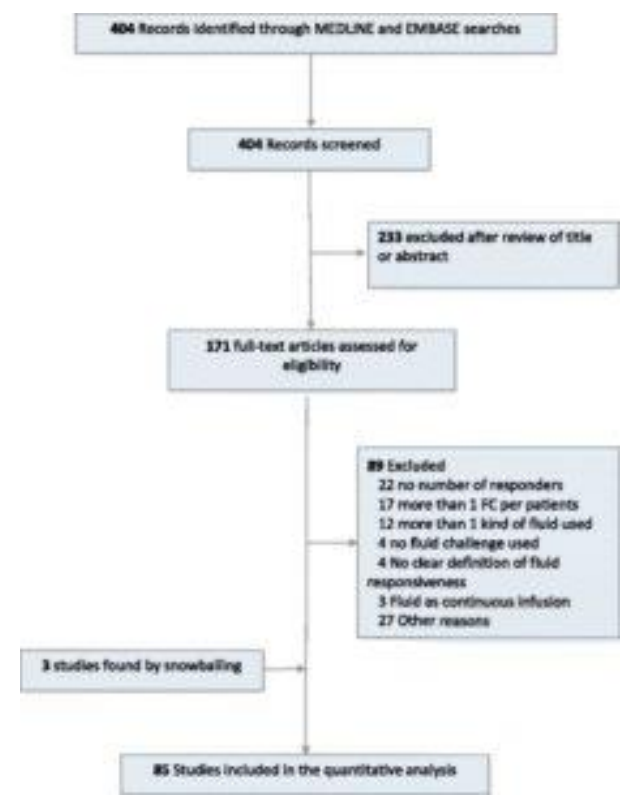

Fig. 1. Flowchart of selection process of studies. FC Fluid challenge. (Fuente: Original)

Se excluyeron las revisiones, los informes de casos y los estudios publicados en forma de resumen. También fueron excluidos los estudios con mujeres embarazadas y niños, estudios en los que se realizó más de una carga de volumen en el mismo paciente, los que incluyeron la elevación pasiva de la pierna sin uso de una técnica de exposición a fluidos, en los que se utilizó más de un tipo de fluido, aquellos que empleaban una infusión continua de líquido, y estudios en los que la capacidad de respuesta del fluido se evaluó solo después de un período de 60 minutos o más después de la finalización de la prueba de fluidos.

Se extrajeron los siguientes datos de cada estudio: volumen de líquido utilizado en la prueba de fluidos, duración de la infusión, tipo de líquido utilizado, definición de la sensibilidad a los fluidos, metodología utilizada para la evaluación de la sensibilidad a los fluidos, características de los pacientes incluidos en el estudio, el entorno clínico en el que se realizó el estudio, el número de pacientes incluidos en el estudio y el porcentaje de "respondedores a líquidos". Los datos fueron extraídos de forma independiente por tres autores y verificados por otro autor.

Los datos se examinaron gráficamente y estadísticamente (prueba de ShapiroWilk) para comprender la distribución y la naturaleza de cada variable. Los datos se presentaron como media e IC del 95\% cuando se distribuyen normalmente o como mediana y rango intercuartílico (IQR) para datos no paramétricos.

El resultado primario del estudio fue la diferencia en los promedios de la proporción de respondedores a líquidos (PR).

Los estudios incluidos se agruparon en tres categorías en función del volumen utilizado para la carga de volumen: $<500 \mathrm{ml}, 500 \mathrm{ml} \mathrm{y}>500 \mathrm{ml}$, también se agruparon en tres categorías para la duración de la infusión de fluidos: $<15$ minutos, entre 15 y 30 minutos $\mathrm{y} \geq 30$ minutos. Los tipos de fluido utilizados se agruparon en dos categorías: coloides y cristaloides.

Se realizó un análisis de ANOVA para comparar los promedios y las varianzas entre los grupos utilizando como segunda variable (el entorno del estudio: UCI frente a quirófano), dada la posible fisiopatología diferente de estos dos grupos y el impacto potencial en el PR. 
Los resultados de las pruebas post hoc se informan con la corrección de Bonferroni para comparaciones múltiples. La significancia estadística fue considerada para $\mathrm{p}<0.05$. El análisis estadístico se realizó utilizando el software IBM SPSS Statistics versión 24.

\section{Resultados}

Un total de 363 artículos fueron identificados a través de PubMed, y 163 fueron identificados a través de Embase. Finalmente se analizaron 88 conjuntos de datos extraídos de 85 estudios con un total de 3601 pacientes.

Los parámetros fisiológicos utilizados para evaluar la respuesta del líquido incluyeron índice cardíaco $(47,5 \%)$, gasto cardiaco $(\mathrm{CO})(17,1 \%)$, volumen sistólico (SV) $(11,0 \%)$ e índice de volumen sistólico $(24,3 \%)$.

El incremento desde las mediciones iniciales para considerar que tenían una respuesta positiva a una prueba de administración de fluidos fue del $10 \%$ (25.5\% de los estudios) o $15 \%$ (74.5\% de los estudios). La definición más frecuente de respuesta positiva a la administración de fluidos fue un aumento en el índice cardíaco de al menos $15 \%(n=33[40.2 \%])$. El CO se estimó utilizando varias tecnologías, utilizándose el $\mathrm{CO}$ continuo (PiCCO; PULSION Medical Systems, Feldkirchen, Alemania) con mayor frecuencia $(31,7 \%$ de los estudios), seguido del catéter de la arteria pulmonar (PAC). Hubo un mayor porcentaje de respondedores en los estudios realizados en quirófano (63.4\%, IC 95\% 58.3-68.4) que en la UCI $(51.5 \%$, IC $95 \%$ 48.2-54.8, p $<0.001)$

a/ Según el volumen administrado.
Los volúmenes de fluido administrados para la prueba de fluidos variaron de $<500 \mathrm{ml}(\mathrm{n}=8$ [12.7\%]) a $500 \mathrm{ml}(\mathrm{n}=$ 50 [79.4\%]) y> $500 \mathrm{ml}(\mathrm{n}=5$ [7.9\%]).

Los valores promedios PR estimados fueron 54,4\% (IC 95\% 46,9-62,7) entre los pacientes que recibieron $<500 \mathrm{ml}$, $57,2 \%$ (IC 95\% 52,9-61,0) entre los pacientes que recibieron $500 \mathrm{ml} \mathrm{y}$ $60,5 \% \quad$ (IC 95\% 35,9-79,2) entre pacientes que reciben $>500 \mathrm{ml}$.

\section{b/ Según la duración de la infusión.}

El tiempo de administración fue $<15$ minutos en 24 estudios (27.3\%), entre 15 y 29 minutos en 26 estudios (29.5\%) $y \geq 30$ minutos en 29 estudios (33\%). Nueve estudios (10.2\%) no informaron la duración de la infusión. Cuando la carga de volumen se administró durante $<15$ minutos, entre 15 y 29 minutos y $>$ 30 minutos, las proporciones de los pacientes considerados respondedores a líquidos fueron $59.2 \%$ (IC 95\% 54.2$64.1), \quad 57.7 \% \quad(95 \%$ CI 52.4-62.4) y $49.9 \% \quad$ (IC $\quad 95 \% \quad 45.6-54$ ) respectivamente. La duración de la infusión de fluidos afecta la proporción de respuestas de fluidos $[\mathrm{F}(2,73)=$ $3.63, \mathrm{p}=0.03]$. La PR a una carga de volumen administrada en $\geq 30$ minutos fue menor que la PR cuando la prueba con fluidos se administró en $<15$ minutos $(\mathrm{p}=0.045)$. La proporción de pacientes que respondieron a la administración de fluidos en $<15$ minutos y entre 15 y 30 minutos fue similar $(\mathrm{p}=1.0)$.

\section{c/ Según el momento de la evaluación.}

La evaluación de la respuesta a una prueba de fluidos fue en el punto de administración $(\mathrm{n}=50[58.1 \%])$, entre 1 y 10 minutos $(n=31[36.8 \%]), o>10$ minutos $(\mathrm{n}=5[5.8 \%])$ después de la finalización de la prueba de fluidos. 
Se evaluó la capacidad de respuesta del fluido en el punto de la administración con un 53.9\% (IC 95\% 49.8-57.7), entre 1 y 10 minutos el $57.7 \%$ (IC 95\% 52.962.7), y > 10 minutos después de la finalización de la prueba con fluido el 52,3\% (IC del 95\%: 32-90,5) de los pacientes tuvieron una respuesta positiva, respectivamente.

El tiempo de evaluación de la respuesta del fluido no afectó la PR [F $(2,80)=$ $0,76, p=0,47]$

\section{Discusión}

Se demostró que la duración de la infusión de fluidos en una carga de volumen tiene una influencia significativa en la capacidad de respuesta del fluido. Otros aspectos de la carga de volumen, incluido el volumen, el tipo de fluido o el tiempo de evaluación, no afectan la proporción de pacientes que responden a los líquidos.

¿Debemos usar coloides o cristaloides? En la carga de volumen, los cristaloides se usaron con mayor frecuencia $(74,0 \%)$, con soluciones equilibradas en la mayoría de los casos (53,3\%) (7-10).

No encontraron ninguna diferencia en PR por el tipo de fluido utilizado para una carga de volumen. Si el tiempo de evaluación de la respuesta del fluido es inmediatamente después de la infusión de fluido o en los primeros minutos, es poco probable que el tipo de fluido cause una diferencia, porque en ambos casos (coloides / cristaloides) es probable que una gran proporción de el volumen infundido permanecerá en el compartimento intravascular. $\mathrm{Si}$ la evaluación de la respuesta a los fluidos se realizara más tarde, sería posible observar algunas diferencias porque teóricamente los coloides permanecen más tiempo en el espacio intravascular que los cristaloides
¿Qué volumen debemos administrar en la carga de volumen?

De acuerdo con un estudio observacional grande reciente (6), el volumen más común de líquido utilizado para la carga de volumen fue de $500 \mathrm{ml}$. Sin embargo, hubo una variabilidad significativa en el volumen de fluido utilizado. El volumen total de líquido administrado para determinar la capacidad de respuesta del fluido varía ampliamente, de 4 a $20 \mathrm{ml} / \mathrm{kg}$ o de 100 a $1000 \mathrm{ml}$.

¿Tiempo que debe durar la administración del líquido? Los resultados de este metaanálisis sugieren que la duración de la infusión de fluidos tiene un efecto significativo en los respondedores a líquidos. Un tiempo de infusión $<30$ minutos es más efectivo para detectar respondedores a líquidos que tiempos de infusión $>30$ minutos.

¿Qué importancia tiene el tiempo en el que se realiza la evaluación? El tiempo de evaluación de una carga de volumen no tiene un impacto significativo en la detección de una respuesta positiva. Esto está en contraste con trabajos previos (11) en el que el efecto hemodinámico de una prueba de fluido con cristaloide de 250 $\mathrm{ml}$ se disipó casi por completo después de 10 minutos desde el final de la exposición a los líquidos.

¿Cómo debemos monitorizar la respuesta a la carga de volumen? En este metaanálisis, muchos estudios utilizaron PAC como método para estimar el $\mathrm{CO}$, que no puede detectar con precisión los cambios inmediatos en SV.

En los estudios observacionales retrospectivos, los datos presentados deben interpretarse en el contexto de sus limitaciones. Destacar la heterogeneidad 
en la práctica de esta técnica comúnmente aplicada.

\section{Conclusiones}

La proporción de pacientes que responden a la administración de fluidos depende de las particularidades de la técnica utilizada. Una infusión rápida de volumen de líquido aumenta la proporción de pacientes con una respuesta positiva. Sin embargo, el tipo y el volumen de líquido o el tiempo de evaluación no parecen tener ningún efecto en la detección de los que responden a los líquidos. Este estudio destaca que la estandarización de la técnica de exposición a fluidos es necesaria para la contextualización de los datos de los ensayos clínicos y el manejo del paciente.

\section{Bibliografía}

1.- Myburgh JA, Mythen MG. Resuscitation fluids. N Engl J Med. 2013;369(25): 2462-3. (ubMed) (ㅁML) $(\underline{\text { PDF }})$

2.- Cecconi M, Parsons AK, Rhodes A. What is a fluid challenge? Curr Opin Crit Care. 2011;17(3):290-5. (PubMed)

3.- Patterson SW, Starling EH. On the mechanical factors which determine the output of the ventricles. J Physiol. 1914;48(5):357-79. ( $\underline{\text { PubMed) }}$ (HTML) (DF1) (DF2)

4.- Cecconi M, Dawson D, Grounds RM, Rhodes A. Lithium dilution cardiac output measurement in the critically ill patient: determination of precision of the technique. Intensive Care Med. 2009;35(3):498-504. (PubMed)

5.- Squara P, Cecconi M, Rhodes A, Singer M, Chiche JD. Tracking changes in cardiac output: methodological considerations for the validation of monitoring devices. Intensive Care Med. 2009;35(10):1801-8. (PubMed)
6.- Cecconi M, Hofer C, Teboul JL, Pettila V, Wilkman E, Molnar Z, et al. Fluid challenges in intensive care: the FENICE study: A global inception cohort study. Intensive Care Med.

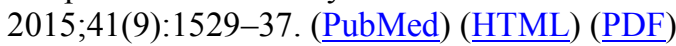

7.- Perner A, Haase N, Guttormsen AB, Tenhunen J, Klemenzson G, Aneman A, et al. Hydroxyethyl starch 130/0. 42 versus Ringer's acetate in severe sepsis. N Engl J Med. 2012;367(2):124-34. (ubMed) (

8.- Myburgh JA, Finfer S, Bellomo R, Billot L, Cass A, Gattas D, et al. Hydroxyethyl starch or saline for fluid resuscitation in intensive care. $\mathrm{N}$ Engl J Med. 2012;367(20):1901-11. (PubMed) ( $\underline{\text { HTML) }}$ (PDF)

9.- Gattas DJ, Dan A, Myburgh J, Billot L, Lo $\mathrm{S}$, Finfer S, Committee CM. Fluid resuscitation with 6\% hydroxyethyl starch (130/0.4 and $130 / 0.42)$ in acutely ill patients: systematic review of effects on mortality and treatment with renal replacement therapy. Intensive Care Med. 2013;39(4):558-68. (PubMed)

10.- Reinhart K, Perner A, Sprung CL, Jaeschke R, Schortgen F, Johan Groeneveld AB, European Society of Intensive Care $M$. Consensus statement of the ESICM task force on colloid volume therapy in critically ill patients. Intensive Care Med. 2012;38(3):36883. ( PubMed)

11.- Aya HD, Ster IC, Fletcher N, Grounds RM, Rhodes A, Cecconi M. Pharmacodynamic analysis of a fluid challenge. Crit Care Med. 2016;44(5):880-91. (PubMed)

Correspondencia al autor

Juan Pedro Macías Pingarrón

jpmaciasp@gmail.com

Servicio de Anestesiología y Reanimación. Complejo Hospitalario Universitario de Badajoz.

Sección de Cuidados Intensivos de la SEDAR

Aceptado para blog en febrero de 2018 